\title{
Aptamer-based and DNAzyme-linked colorimetric detection of cancer cells
}

\author{
Xiaoli Zhu' ${ }^{1}$, Ya Cao ${ }^{2}$, Zhiqiang Liang ${ }^{1}$, Genxi $\mathrm{Li}^{1,2} \bowtie$ \\ ${ }^{1}$ Laboratory of Biosensing Technology, School of Life Science, Shanghai University, Shanghai 200444, China \\ 2 Department of Biochemistry and National Key Laboratory of Pharmaceutical Biotechnology, Nanjing University, Nanjing \\ 210093, China \\ $\triangle$ Correspondence: genxili@nju.edu.cn \\ Received August 11, 2010 Accepted September 6, 2010
}

\begin{abstract}
This paper reports a novel method to detect human leukemic lymphoblasts (CCRF-CEM cells). While the aptamer of the cancer cells was employed as the recognition element to target cancer cells, peroxidaseactive DNAzyme was used as the sensing element to produce catalysis-induced colorimetric signals. The elegant architecture integrating the aptamer and DNAzyme made it feasible to detect cancer cells easily and rapidly by the color change of the substrate for DNAzyme. Experimental results showed that 500 cells can well indicate the cancer, while as control, 250,000 Islet Island Beta cells only show tiny signals, suggesting that the method proposed in this paper has considerable sensitivity and selectivity. Furthermore, since it does not require expensive apparatus, or modification or label of DNA chains, the method we present here is also costeffective and conveniently operated, implying potential applications in future cancer diagnosis.
\end{abstract}

KEYWORDS CCRF-CEM acute leukemia cells, aptamer, DNAzyme, colorimetry

\section{INTRODUCTION}

Cancer is one of the most critical threats to the health of human beings (Pui and Evans, 1998; Kroemer and Pouyssegur, 2008). Early diagnosis and timely treatment have been verified as the most effective ways to improve survival rate at present (Ghossein and Bhattacharya, 2000; ParedesAguilera et al., 2001). Although various techniques have been developed for cancer diagnosis (Luo et al., 2003; Padgett et al., 2003; Espina et al., 2005), most of them still cannot meet the demands of "early diagnosis".
Cancer first takes place at molecular level, so the method targeting tumor-associated antigens is one of the most promising approaches to pursue "early diagnosis", and some antibody-based biosensors are reported for cancer diagnosis at molecular level (Shao et al., 2008; Weerkamp et al., 2009). However, suffering from the high cost and low screening efficiency, it is still far away to be a universal technique. Aptamer, since the first description in 1990, has been considered as a unique substitute for antibody in the fields of biosensors and therapeusis (Ellington and Szostak, 1990; Tuerk and Gold, 1990; Osborne and Ellington, 1997; Shangguan et al., 2006). Aptamer-based biosensors not only exhibit desirable selectivity, specificity and affinity, but also show distinguished advantages (Famulok et al., 2000; Xiao et al., 2005), allowing them to be a senior method for biosensing and diagnostic applications. In the late decade, aptamers have been broadly used in the fabrication of biosensor for the detection of ions (Liu et al., 2009), micromolecules (Baker et al., 2006) and proteins (Degefa and Kwak, 2008). Recently, Herr et al. (2006) made use of aptamer to target cancer cells. From then on, a variety of aptamer-based assays for cancer cells have been developed with different measuring techniques, including fluorescence (Chen et al., 2009), colorimetry (Herr et al., 2006; Smith et al., 2007; Medley et al., 2008) and electrochemistry (Pan et al., 2009). These efforts provide a novel concept for the early cancer diagnosis.

Though the previous reported aptamer-based biosensors for cancer cell detection have shown advantages of simplicity, rapidness and cost-efficiency over traditional methods (Herr et al., 2006; Smith et al., 2007; Medley et al., 2008; Chen et al., 2009; Pan et al., 2009), there is still huge potential for further improvement. Here, by combining aptamer and DNAzyme together, we developed an aptamer-based and DNAzyme-linked colorimetric biosensor for the detection of CCRF-CEM acute leukemia cells. This method may also 
avoid the modification of DNA and the use of labels, which can dramatically increase the simplicity and cost-efficiency of cancer cell detection.

\section{RESULTS AND DISCUSSION}

Scheme 1 illustrates the colorimetric assay strategy for the detection of cancer cells in this study. Two finely designed single-stranded DNA (ssDNA) chains are essential for this method. As shown in the scheme, both ssDNA-1 and ssDNA-2 contain two interlaced functional regions. While ssDNA-1 consists of an aptamer region targeting CCRF-CEM cells (green and sky-blue) and a linker region (sky-blue and blue), ssDNA-2 has a horseradish-peroxidase-mimicking DNAzyme unit (orange and yellow) and a linker region (yellow and sky-blue), which is complementary to the linker region of ssDNA-1. In the presence of target cells, ssDNA-1 will bind to the cell surface and then be removed from the system under centrifugation. Therefore, the ssDNA-2 supplemented to supernate will be free from the hybridization with ssDNA-1 and form peroxidase-active G-quadruplex with hemin. Subsequently, the G-quadruplex catalyzes $\mathrm{H}_{2} \mathrm{O}_{2}$ mediated oxidation of 2,2'-azino-bis(3-ethylbenzothiazoline)6-sulfonate $\left(\mathrm{ABTS}^{2-}\right)$ to $\mathrm{ABTS}{ }^{-}$, presenting color change from light green to dark green, and the color change can be easily monitored by both spectrophotometer and naked eyes. On the other hand, in the absence of target cells, ssDNA-1 will remain in the supernate to hybridize with ssDNA-2, forming double-stranded DNA (dsDNA). The rigid structure of dsDNA blocks the formation of peroxidase-active G-quadruplex, and thereby no catalytic reaction will occur. Here, the peroxidaseactive DNAzyme not only facilitates the fabrication of labelfree and unmodified architecture, but also allows the amplification of the sensing signals.
Because the colorimetric signals are derived from the catalytic reaction of the DNAzyme (peroxidase-active Gquadruplex formed by ssDNA-2 and hemin), assay for the enzymatic activity of DNAzyme was first studied so as to obtain a largest signal production. As shown in Fig. 1A, the catalysis-induced absorbance change of $\mathrm{ABTS}^{2-}$ at $414 \mathrm{~nm}$ increased along with the concentration of SSDNA-2, and a concentration of $50 \mathrm{nM}$ ssDNA-2 was enough to produce

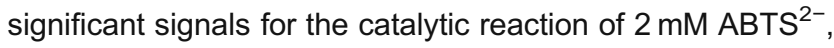
while a higher concentration of ssDNA had little effect on enhancing catalytic activity. Therefore, we adopted $50 \mathrm{nM}$ ssDNA-2 for the following experiments.

Fig. 1B shows the time-dependent catalytic curves of DNAzyme with no ssDNA-2, $50 \mathrm{nM}$ ssDNA-2, or $50 \mathrm{nM}$ ssDNA-2 that was pre-incubated with $50 \mathrm{nM}$ ssDNA-1 to form dsDNA. It can be observed that a typical catalytic reaction was achieved in the presence of SSDNA-2. As control, if only hemin was present, no significant absorbance change could be detected, suggesting that the reaction is catalyzed by the complex of ssDNA-2 and hemin. Meanwhile, if ssDNA-2 hybridized with ssDNA-1, no peroxidase-active Gquadruplex formed, so the typical catalytic reaction could not occur again.

As mentioned above, for the detection of CCRF-CEM cells, the aptamer region of sSDNA-1 will bind to the surface of the cancer cells and SSDNA-1 will be removed from the assay system under centrifugation; consequently, ssDNA-2 cannot hybridize with ssDNA-1. Therefore, the optional time for the cancer cells to incubate with the aptamer-containing ssDNA-1 should be determined. As shown in Fig. 2, 30 min was the best incubation time to produce the best signal. Meanwhile, it is interesting that the elongation of the incubating time however did not result in a higher absorbance of ABTS ${ }^{-}$. The reason is still unknown and will be on our schedule for

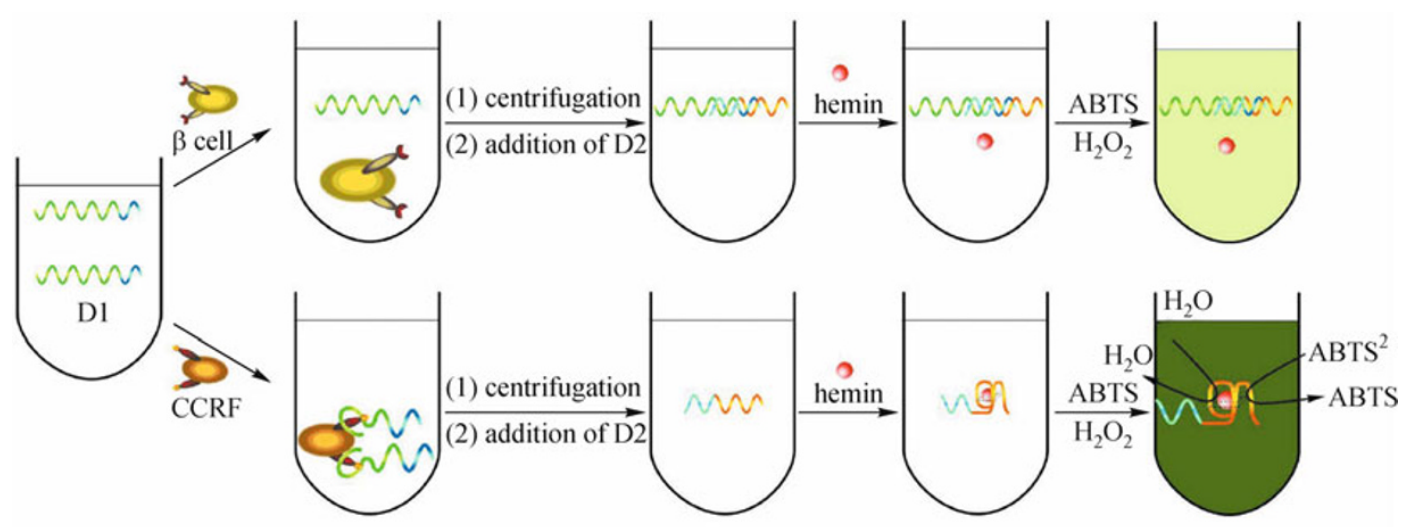

D1 5'-ATCTA ACTGC TGCGC CGCCG GGAAA ATACT GTACG GTTAG ACCCA ACCC

D2 5'-TGGGT AGGGC GGGTT GGGTC TAACC GTACA GTA

Scheme 1. Schematic illustration of the colorimetric assay strategy for the detection of cancer cells. D1, D2, CCRF, and $\beta$ cell are short for ssDNA-1, ssDNA-2, CCRF-CEM cell and Islet Beta cell, respectively. 

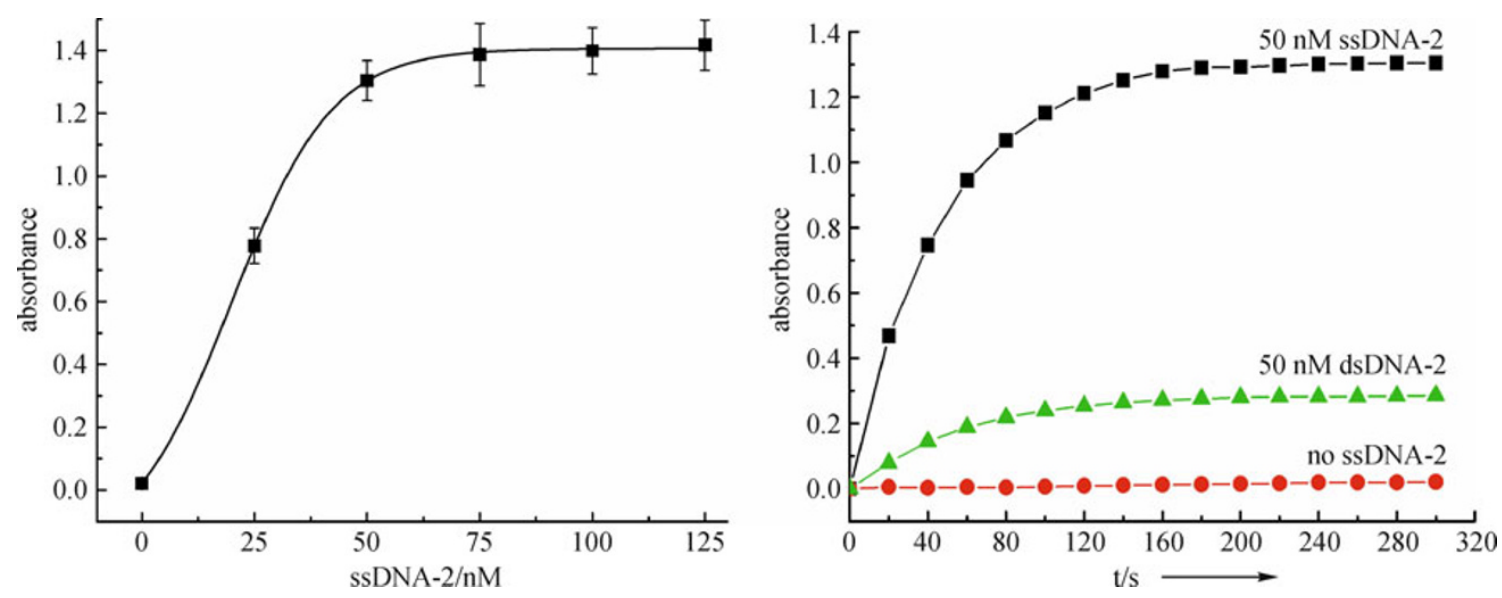

Figure 1. Absorbance of the oxidized $\mathrm{ABTS}^{2-}\left(\mathrm{ABTS}{ }^{-}\right.$) and the time-dependent catalytic curves of DNAzyme. (A) Absorbance of the oxidized ABTS $^{2-}\left(\right.$ ABTS $^{-}$) at $414 \mathrm{~nm}$ vs. the concentration of ssDNA-2. Reaction time is fixed at $300 \mathrm{~s}$. (B) Timedependent catalytic curves of peroxidase-active G-quadruplex in the presence of no ssDNA-2, 50 nM ssDNA-2, or 50 nM ssDNA-2 that was pre-incubated with $50 \mathrm{nM}$ ssDNA-1 for $2 \mathrm{~h}$ to form dsDNA. ABTS ${ }^{2-}$ concentration is $2 \mathrm{mM}$.

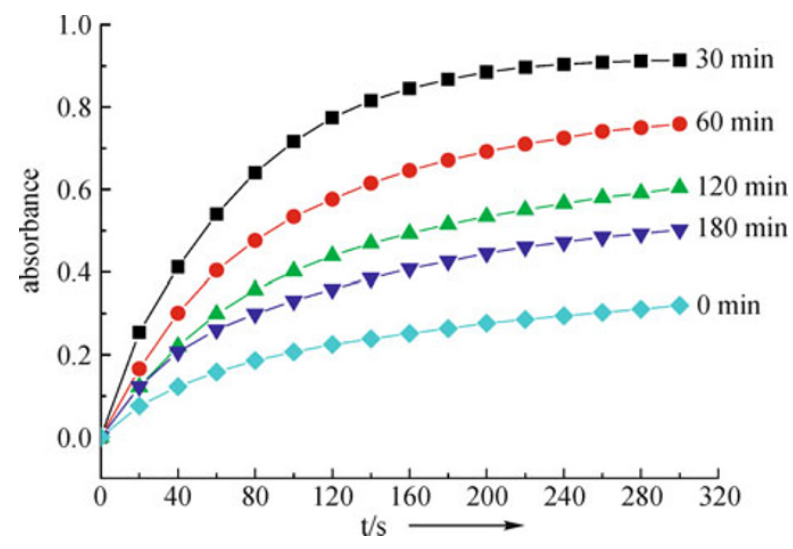

Figure 2. Time-dependent catalytic response for the detection of 250,000 CCRF-CEM cells. The incubating time of CCRF-CEM cells with the aptamer-contained SSDNA-1 is $0 \mathrm{~min}, 30 \mathrm{~min}, 60 \mathrm{~min}, 120 \mathrm{~min}$, and $180 \mathrm{~min}$, respectively.

further studies. Nevertheless, since the CCRF-CEM cells can successfully lead to significant signal-on colorimetric results, detection of the cancer cells can be accomplished.

We then used this aptamer-based and DNAzyme-linked method to detect different amounts of CCRF-CEM cells, using Islet Beta cells as control. It can be observed from Fig. 3 that 500 CCRF-CEM cells was enough to present discriminated curve from that without cells, and the absorbance increased along with the cell amounts. Thus, the colorimetric signals have a positive relationship with the amount of cells, suggesting the potential application of this novel method in the quantitative detection of cancer cells. Meanwhile, the results of the control experiments revealed that this method also displayed a considerable selectivity. Furthermore, the

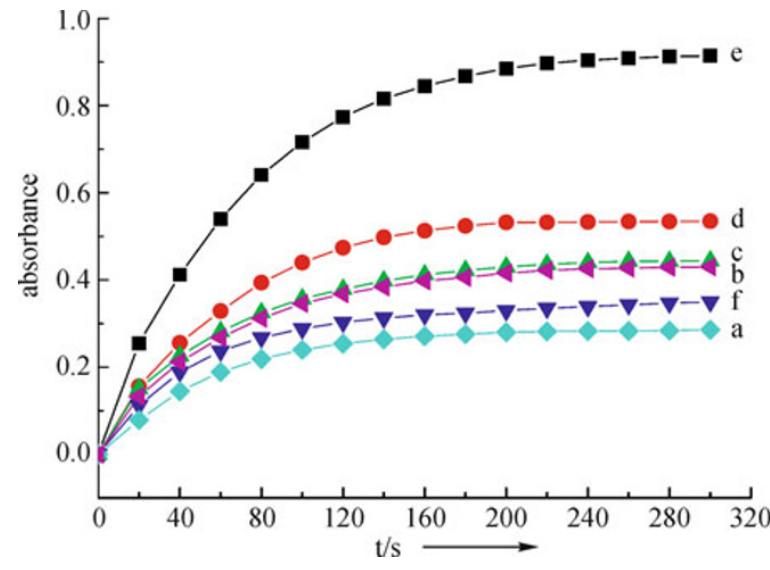

Figure 3. Time-dependent catalytic response for the detecting different amount of CCRF-CEM cells. From (a) to (e): $0,500,2,500,25,000$ and 250,000 CCRF-CEM cells. Curve f: 250,000 Islet Beta cells.

detection limits of recent reports were in a level of $1 \times 10^{3}$ cells (He et al., 2009; Pan et al., 2009); benefiting from the DNAzyme-linked amplification, we were able to detect cancer cells in $1 \times 10^{2}$ level.

\section{CONCLUSION}

In summary, we developed a novel method to colorimetrically detect cancer cells. While the aptamer of the targeting CCRFCEM cells was adopted as recognition element, peroxidaseactive DNAzyme that can catalyze the oxidation of $\mathrm{ABTS}^{2-}$ to the colored ABTS ${ }^{-}$was used to produce the colorimetric signals. The ingenious strategy to relate the recognition with catalysis makes the architecture effectively feed back positive 
colorimetric signals upon the presence of CCRF-CEM cells. Results showed that 500 CCRF-CEM cells were enough to result in recognizable colorimetric signals, and Islet Beta cells that were not the target of the aptamer produced nearly no signal. The method is not only simple, fast and cost-effective, but also sensitive and selective, since, for comparison, immunophenotypic analysis often requires multiple labeled antibody probes, increasing both the complexity and the cost of the method, while PCR-based methods otherwise require prolonged RNA isolation steps before analysis, which is also an arduous task. Moreover, the color change of the test solution, which can be recognized by naked eye, makes the analysis to be more simply and easily operated. Since this proposed method can be also extended for the detection of some other cancer cells by using corresponding aptamers, e.g., Ramos cell-targeted aptamer, it may have great potential for the early diagnosis of cancer.

\section{MATERIALS AND METHODS}

\section{Chemicals}

Hemin and $\mathrm{ABTS}^{2-}$ were purchased from Sigma-Aldrich and used without further purification. A hemin stock solution was prepared with DMSO and stored in dark at $-20^{\circ} \mathrm{C}$. ssDNA chains (Guaranteed Oligos, HPLC-purified, ssDNA-1: 5'-ATCTAACTGCTGCGCCGCCGGGAAAATACTGTACGGTTAGACCCAACCC-3', ssDNA-2: 5'TGGGTAGGGCGGGTTGGGTCTAACCGTACAGTA-3') were custom-ordered from Invitrogen. All solutions were prepared by double-distilled water, which was purified with a Milli-Q purification system (Branstead, USA) to a specific resistance of $>18 \mathrm{M} \Omega \mathrm{cm}$.

\section{Cell culture and preparation}

CCRF-CEM cells and Islet Beta cells were obtained from the Committee on Type Culture Collection of Chinese Academy of Sciences. The cells were cultured in RPMI 1640 medium supplemented with $10 \%$ fetal bovine serum (FBS). Before use, the cell density was determined by hemocytometer counting. Briefly, the suspension of a cell sample was first introduced into one of the Vshaped wells with a pipet, and the cells in selected squares were systematically counted under microscope. The cell density could be calculated according to the ratio of the total cell number in selected squares to the total square volume. Then, the cells dispersed in RPMI cell media buffer were centrifuged at $920 \mathrm{rpm}$ for $5 \mathrm{~min}$, and redispersed in phosphate buffered solution $(\mathrm{pH} 6.2)$ for the following experiments. During all the experiments, the cells were kept in an ice bath at $4^{\circ} \mathrm{C}$ to prevent internalization of DNA chains into cells.

\section{Colorimetric analysis}

CCRF-CEM cells or Islet Beta cells were first incubated with $100 \mathrm{nM}$ SSDNA-1, which contained an aptamer region of the CCRF-CEM cells. After a certain period of incubation, the samples were centrifuged at $920 \mathrm{rpm}$ for $5 \mathrm{~min}$ to remove the cells, and the aptamer-contained oligonucleotides were also removed out of the solution if CCRF-CEM cells were used. Then, ssDNA-2 (100 nM) containing a DNAzyme unit was added to the supernate, which was subsequently heated to $90^{\circ} \mathrm{C}$ and cooled down to room temperature in $2 \mathrm{~h}$. After that, the sample was mixed $(1: 1, v / v)$ with a solution of hemin (100 nM in 40KT buffer: $50 \mathrm{mM} \mathrm{pH} \mathrm{6.2} \mathrm{Tris-HCl} \mathrm{buffer} \mathrm{solution}$ containing $40 \mathrm{mM} \mathrm{KCl}, 0.05 \%(w / v)$ Trition X-100, and $100 \mathrm{mM}$ MES) and incubated for one hour to form G-quadruplex assembly. ABTS ${ }^{2-}$ (2 $\mathrm{mM})$ and $\mathrm{H}_{2} \mathrm{O}_{2}(2 \mathrm{mM})$ as the substrates of the peroxidase-active G-quadruplex were then added. The catalysis was monitored by measuring the biocatalyzed oxidation of $\mathrm{ABTS}^{2-}$ at a wavelength of $414 \mathrm{~nm}$. Absorbance measurements were performed by using a Shimadzu UV-2450 UV/Vis spectrophotometer. All the experiments were conduced for at least four times, and the data presented were averages from at least four independent experiments. The relative standard deviation (RSD) of every data point was lower than $5.5 \%$, suggesting a good reproducibility.

\section{ACKNOWLEDGEMENTS}

This work is supported by the National Science Fund for Distinguished Young Scholars (Grant No. 20925520).

\section{ABBREVIATIONS}

ABTS $^{2-}$, 2,2'-azino-bis(3-ethylbenzothiazoline)-6-sulfonate; CCRFCEM cells, human leukemic lymphoblasts; dsDNA, double-stranded DNA; FBS, fetal bovine serum; ssDNA, single-stranded DNA

\section{REFERENCES}

Baker, B.R., Lai, R.Y., Wood, M.S., Doctor, E.H., Heeger, A.J., and Plaxco, K.W. (2006). An electronic, aptamer-based small-molecule sensor for the rapid, label-free detection of cocaine in adulterated samples and biological fluids. J Am Chem Soc 128, 3138-3139.

Chen, X., Estévez, M.C., Zhu, Z., Huang, Y.F., Chen, Y., Wang, L., and Tan, W. (2009). Using aptamer-conjugated fluorescence resonance energy transfer nanoparticles for multiplexed cancer cell monitoring. Anal Chem 81, 7009-7014.

Degefa, T.H., and Kwak, J. (2008). Label-free aptasensor for plateletderived growth factor (PDGF) protein. Anal Chim Acta 613, 163-168.

Ellington, A.D., and Szostak,J.W. (1990). In vitro selection of RNA molecules that bind specific ligands. Nature 346, 818-822.

Espina, V., Geho, D., Mehta, A.I., Petricoin, E.F., Liotta, L.A., and Rosenblatt, K.P. (2005). Pathology of the future: molecular profiling for targeted therapy. Cancer Invest 23, 36- 46.

Famulok, M., Mayer, G., and Blind, M. (2000). Nucleic acid aptamersfrom selection in vitro to applications in vivo. Acc Chem Res 33, 591-599.

Ghossein, R.A., and Bhattacharya, S. (2000). Molecular detection and characterisation of circulating tumour cells and micrometastases in solid tumours. Eur J Cancer 36, 1681-1694.

He, F., Shen, Q., Jiang, H., Zhou, J., Cheng, J., Guo, D., Li, Q., Wang, X., Fu, D., and Chen, B. (2009). Rapid identification and high sensitive detection of cancer cells on the gold nanoparticle interface by combined contact angle and electrochemical measurements. Talanta 77, 1009-1014.

Herr, J.K., Smith, J.E., Medley, C.D., Shangguan, D., and Tan, W. (2006). Aptamer-conjugated nanoparticles for selective collection and detection of cancer cells. Anal Chem 78, 2918-2924. 
Kroemer, G., and Pouyssegur, J. (2008). Tumor cell metabolism: cancer's Achilles' heel. Cancer Cell 13, 472-482.

Liu, C.W., Huang, C.C., and Chang, H.T. (2009). Highly selective DNA-based sensor for lead(II) and mercury(II) ions. Anal Chem 81, 2383-2387.

Luo, J., Isaacs, W.B., Trent, J.M., and Duggan, D.J. (2003). Looking beyond morphology: cancer gene expression profiling using DNA microarrays. Cancer Invest 21, 937-949.

Medley, C.D., Smith, J.E., Tang, Z., Wu, Y., Bamrungsap S., and Tan, W. (2008). Gold nanoparticle-based colorimetric assay for the direct detection of cancerous cells. Anal Chem 80, 1067-1072.

Osborne, S.E., and Ellington, A.D. (1997). Nucleic acid selection and the challenge of combinatorial chemistry. Chem Rev 97, 349-370.

Padgett, J.K., Parlette, H.L., and English, J.C. (2003). A diagnosis of chronic lymphocytic leukemia prompted by cutaneous lymphocytic infiltrates present in mohs micrographic surgery frozen sections. Dermatol Surg 29, 769-771.

Pan, C., Guo, M., Nie, Z., Xiao, X., and Yao, S. (2009). Aptamerbased electrochemical sensor for label-free recognition and detection of cancer cells. Electroanalysis 21, 1321-1326.

Paredes-Aguilera, R., Romero-Guzman, L., Lopez-Santiago, N., Burbano-Ceron, L., Camacho-Del Monte, O., and Nieto-Martinez, S. (2001). Flow cytometric analysis of cell-surface and intracellular antigens in the diagnosis of acute leukemia. Am J Hematol 68, 69-74.
Pui, C.H., and Evans, W.E. (1998). Acute lymphoblastic leukemia. N Engl J Med 339, 605-615.

Shangguan, D., Li, Y., Tang, Z., Cao, Z.C., Chen, H.W., Mallikaratchy, P., Sefah, K., Yang, C.J., and Tan, W. (2006). Aptamers evolved from live cells as effective molecular probes for cancer study. Proc Natl Acad Sci U S A 103, 11838-11843.

Shao, N., Wickstrom, E., and Panchapakesan, B. (2008). Nanotubeantibody biosensor arrays for the detection of circulating breast cancer cells. Nanotechnology 19, 465101.

Smith, J.E., Medley, C.D., Tang, Z., Shangguan, D., Lofton, C., and Tan, W. (2007). Aptamer-conjugated nanoparticles for the collection and detection of multiple cancer cells. Anal Chem 79, 3075-3082.

Tuerk, C., and Gold, L. (1990). Systematic evolution of ligands by exponential enrichment: RNA ligands to bacteriophage T4 DNA polymerase. Science 249, 505-510.

Weerkamp, F., Dekking, E., Ng, Y.Y., van der Velden, V.H.J., Wai, H., Böttcher, S., Brüggemann, M., van der Sluijs, A.J., Koning, A., Boeckx, N., et al.(2009). Flow cytometric immunobead assay for the detection of BCR-ABL fusion proteins in leukemia patients. Leukemia 23, 1106-1117.

Xiao, Y., Piorek, B.D., Plaxco, K.W., and Heeger, A.J. (2005). A reagentless signal-on architecture for electronic, aptamer-based sensors via target-induced strand displacement. J Am Chem Soc 127, 17990-17991. 